\title{
Unraveled Material
}

National Cancer Institute

\section{Source}

National Cancer Institute. Unraveled Material. NCI Thesaurus. Code C62811.

Problem due to the device's undesired unravelling of material (e.g. disentangled, unwound etc.). 